\title{
De l'apprentissage à la formation. Pour une nouvelle psychopédagogie des adultes
}

\author{
From learning to training. Towards a new conceptual framework for \\ adult education theory
}

\author{
Philippe Carré \\ philippe.carre@u-paris10.fr \\ Université Paris-Ouest-Nanterre-La Défense, CREF
}

Le micromonde de la formation diffuse et entretient un contresens psychologique et pédagogique en subsumant le processus d'apprentissage des adultes sous la pratique de la formation. Partant de constats établis au plan international, nous cherchons à confirmer cette priorité à l'" apprendre » dans la compréhension des faits de formation et la recherche d'efficience des pratiques. Pour ce faire, cet article posera un bref rappel des définitions, puis l'hypothèse d'une "erreur pédagogique fondamentale " dans les conceptions courantes et, parfois, expertes, de la formation des adultes. À travers un aperçu des grandes conceptions de l'apprentissage qui traversent les milieux de la formation, nous mentionnerons ensuite en quoi ces concepts et théories contribuent à la rénovation des visions de la formation et autorisent à en mieux penser l'efficacité, par-delà effets de mode et emballements techniques ou pédagogiques. La question proprement pédagogique de l'intervention "facilitante" sera enfin ébauchée avant de conclure par une proposition de cadre conceptuel pour une psychopédagogie des adultes renouvelée.

Mots-clés (TESE) : formation des adultes, théorie d'apprentissage, pédagogie, apprenant adulte, apprentissage tout au long de la vie.

The microworld of adult education and training diffuses and maintains a psychological misinterpretation by subsuming the process of learning under the practice of training. On the basis of international literature, we seek to confirm this "learning priority" in the understanding and improvement of adult education. The article starts with a short recall of definitions, then presents the assumption that a "fundamental teaching error" operates in the current and, sometimes, expert, views of adult training. Through an outline of the main learning paradigms that flow through the adult learning literature, we then mention how these theories help renovate our visions of learning and development theory, beyond the short-lived effects of technological and/or pedagogical vogues. The question of intervention will be finally outlined before we conclude with a proposal for a renewed conceptual framework for adult education.

Keywords (TESE): adult education, learning theorie, educational theory, adult learner, lifelong learning.

Depuis la date symbolique de 1971 et l'institutionnalisation massive de la formation professionnelle continue (FPC) en France, plusieurs évolutions majeures amènent aujourd'hui à 
reconsidérer les rapports entre les notions de formation et d'apprentissage des adultes. Renouant avec les principes de l'éducation permanente, mais significativement rétrécie aux finalités du travail et de l'emploi, une culture de l'apprentissage tout au long de la vie (lifelong learning) vient peu à peu concurrencer la culture ancienne de la formation (training). L'évolution récente du droit dans le domaine de la formation signe ainsi la primauté graduelle des dynamiques individuelles d'apprentissage avec les lois sur la VAE (2002), le droit individuel à la formation (2004), l'orientation tout au long de la vie (2009) et la consécration d'un « compte personnel de formation » (2014). Les grands organismes internationaux, de l'Union européenne à l'UNESCO et du Cedefop à l'OCDE, proclament à l'unisson depuis vingt ans la nécessité absolue du développement de cet apprentissage quotidien et permanent, formel et informel, comme ingrédient essentiel des «sociétés cognitives» en émergence (Carré, 2005). De tous côtés, les décideurs promeuvent, invitent et parfois imposent, une logique d'apprentissage dans, par et pour l'activité professionnelle. Le développement des formations en alternance, l'essor des formes diverses d'analyse des pratiques, de la formation en situation de travail et l'intérêt croissant pour les apprentissages professionnels informels sont autant de balises qui pointent la direction de cette mutation. L'explosion technologique, en particulier sous ses formes les plus récentes, déclenche et entretient l'évidence chaque jour plus aiguë de formes variées d'autoformation «numérique». Les institutions, de l'entreprise à la région, sont appelées à devenir «apprenantes». La vie quotidienne elle-même est aujourd'hui amplement reconnue comme source des apprentissages les plus variés, que ce soit au travail, dans les loisirs, les activités citoyennes ou la famille (Brougère \& Ullmann, 2009). Ce tournant de l'apprentissage permanent, «tout au long» et «tout au large » de la vie, impose de porter un regard critique et solidement adossé aux connaissances accumulées sur l'acte d'apprendre et la formation pour faire face à ces défis multiples adressés aux sujets sociaux, qu'ils soient étudiants, salariés, parents, éducateurs ou professionnels de la formation. Le recours à la recherche est ici nécessaire.

Sur ces bases, cet article assumera le parti pris selon lequel une partie du monde de la formation diffuse et entretient, encore aujourd'hui, un contresens psychologique et pédagogique en subsumant le processus d'apprentissage des adultes sous la pratique de la formation. Partant de constats établis sur ces questions au plan international, nous chercherons à confirmer cette priorité à l'«apprendre » dans la compréhension des faits de formation d'adultes et la recherche d'efficience des pratiques. Pour ce faire, cet article posera un bref rappel des définitions, puis l'hypothèse d'une «erreur pédagogique fondamentale» dans les conceptions courantes et, parfois, expertes, de la formation des adultes. À travers un aperçu des grandes conceptions de l'apprentissage qui traversent les milieux de la formation, nous mentionnerons ensuite en quoi ces concepts et théories contribuent à la rénovation des visions de la formation et autorisent à en 
mieux penser l'efficacité, par-delà effets de mode et emballements techniques ou pédagogiques éphémères. La question proprement pédagogique de l'intervention «facilitante» sera enfin ébauchée avant de conclure par une proposition de cadre conceptuel pour une psychopédagogie des adultes renouvelée.

\section{L'erreur pédagogique fondamentale}

\section{Apprentissage et formation ne sont pas synonymes}

La première des difficultés qui se présente à qui souhaite analyser les rapports entre apprentissage et formation est que ces termes sont souvent utilisés sans être définis, tant leur signification paraît évidente. On les pense facilement synonymes : selon cette psychopédagogie sommaire, la formation, bien faite, mènerait automatiquement à l'apprentissage, et apprendre supposerait d'être en formation. Or les choses sont loin d'être aussi évidentes. Dans les termes de Vermersch (2012, p. 244) :

Le sens ne peut avoir d'effets que de manière médiée : par la compréhension que le destinataire en a et par la disposition (consentement, refus, indifférence) qu'il manifeste à suivre ce qu’il comprend. Cette médiation est clairement illustrée, par exemple, par la distinction basique entre enseigner et apprendre. Le premier acte est celui du maître, mais il ne produit pas le second, il n'en crée que les conditions favorisantes. Le second, l'acte d'apprendre, est l'affaire de l'apprenant. L'acte d'enseigner ne produit une activité d'apprentissage qu'à la mesure de l'activité de l'élève qui n'est que suscitée par les paroles du maitre.

Le terme d'apprentissage décrit le processus psychologique, interne au sujet bien que toujours socialement situé, qui mène à la transformation durable de représentations, d'habiletés et d'attitudes, en milieu éducatif formel ou ailleurs. La notion de formation, quant à elle, décrit une intervention sur autrui, en milieu généralement formel, visant le développement des compétences; en ce sens elle ne recouvre qu'une intention de transformation d'autrui. Dès l'abord, une fracture s'instaure entre les deux notions. L'apprentissage est un phénomène intime, une «action sur soi » dont le sujet social est à la fois l'hôte et l'acteur, voire l'auteur. En revanche, la formation est un projet de modification du «formé », une tentative d'action sur l'autre. C'est cette tentative de transformation d'autrui par la formation qui est aujourd'hui largement questionnée. Le constat réitéré du peu d'efficacité de la formation «descendante », les difficultés lancinantes pour en développer l'évaluation, l'impact de tensions budgétaires accrues, l'essoufflement de la formule canonique du stage, les désillusions du e-learning viennent, en un face-à-face explosif, rencontrer la montée en puissance apparemment incontrôlable de l'usage 
des ressources numériques et des manifestations multiples de l'apprentissage informel dans une société aujourd'hui saturée d'informations, y compris expertes. Ce contexte sociopédagogique amène à rappeler trois évidences souvent oubliées (voir figure 1) :

(a) on peut apprendre en dehors de la formation ;

(b) on peut être en formation et ne rien apprendre ;

(c) fort heureusement, il est des situations de formation où l'on apprend.

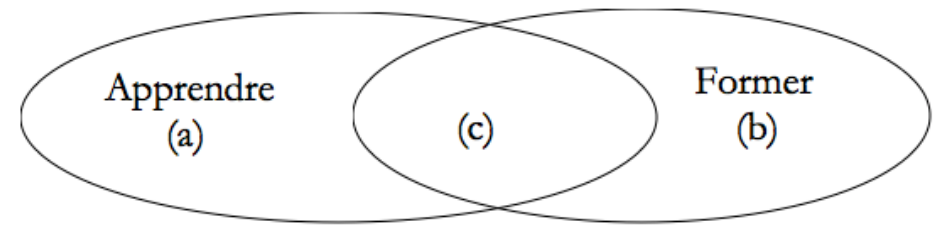

Figure 1. Apprendre et former : deux activités distinctes

Ce décalage entre l'acte d'apprendre, radicalement lié à la problématique singulière du sujet social concerné, et celui de former, dont la responsabilité incombe à un «autre » investi du rôle d'enseignant, d'éducateur ou de formateur, apparaît en effet de plus en plus brutalement à mesure que se multiplient les constats sur la portée des apprentissages informels, expérientiels, autodirigés dans le quotidien du travail et de la vie courante (zone (a) dans la figure 1). On en prendra pour preuves récurrentes les témoignages accumulés de sujets engagés dans des apprentissages multiples en marge de l'école et de la formation (dont une infime partie seulement trouve sa reconnaissance par la VAE ou le retour aux études «légitimes »), que ce soit en situation de travail (Carré \& Charbonnier, 2003) ou dans la vie quotidienne (Nagels \& Carré, 2014).

Les déceptions liées à l'absentéisme, au peu de résultats, aux complexités du transfert et à la faible efficacité des investissements en formation font l'objet de critiques lancinantes et répétitives depuis la fin de l'état de grâce qui a suivi la promulgation de la loi de 1971. Les modalités administratives et comptables de gestion de la formation en entreprise dominent les pratiques, rendant la question de l'efficacité pédagogique et des apprentissages caduque avant même que d'être posée. Ainsi, maint stage de formation ou projet de e-learning, s'il peut produire des effets " collatéraux », par exemple en termes de climat social, d'image d'entreprise ou de communication, ne génère que pas ou très peu d'apprentissages (zone (b) dans la figure 1 ). Les situations de formation «apprenantes» (zone (c) dans la figure 1), fort heureusement nombreuses, concrétisent l'articulation entre logique du sujet apprenant et logique d'intervention (Lameul, Jézégou \& Trollat, 2009). Le célèbre postulat de Schwartz selon lequel un adulte ne se formera que s'il trouve dans la formation une réponse à ses problèmes, dans sa situation, trouve ici une nouvelle légitimité. Cette rencontre souhaitée entre formation et 
apprentissage est loin d'être l'«allant de soi » que présuppose une vision de la formation sommaire, mais répandue.

Comme le résume Enlart (2013), il y a désormais...

un décalage de plus en plus impressionnant entre ce qu'on appelle aujourd'hui formation, mais qui ne permet pas toujours d'apprendre, et les processus d'apprentissage qui sont à l'œuvre et qui permettent de professionnaliser et de développer les compétences [...]. On apprend aussi en formation, mais ce n'est pas toujours là que se passe l'essentiel.

Formation et apprentissage sont donc loin d'être synonymes. L'une des causes, majeure, de ce bilan mitigé de l'impact de la formation sur les apprentissages adultes tient à la force des préconceptions courantes sur la relation entre formation et performance.

\section{La triple illusion de la formation continue}

Tout se passe en effet comme si la formation, de par ses vertus intrinsèques, pouvait automatiquement entraîner la performance (économique, sociale, humaine), par la simple vertu de la transmission de savoirs. Quel que soit le problème posé (augmentation de la productivité d'un service commercial, management d'une unité en situation conflictuelle, niveau insuffisant de compétence des salariés en bureautique ou en langues, modification des normes de sécurité, introduction de nouveaux équipements ou procédures, etc.), le premier réflexe du décideur est de penser « formation » (voir figure 2).

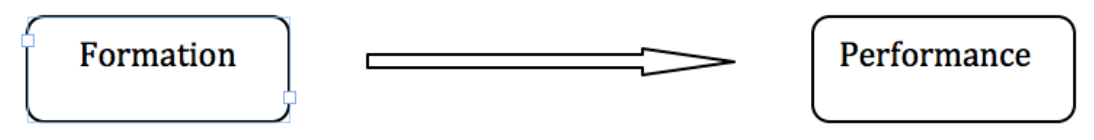

Figure 2. Une fausse évidence

Source : Carré, 2005.

Cette image classique des vertus magiques de la formation fait l'impasse sur trois conditions « intermédiaires » de cette opération (voir figure 3):

- pour être utile, la formation doit permettre des apprentissages. Or il n'est pas prouvé, loin s'en faut, que tout acte de formation entraîne des effets d'apprentissage, et encore moins ceux-là mêmes prévus par le formateur ou l'organisateur ;

- pour se transformer en compétence, l'apprentissage in vitro doit pouvoir être mis à l'épreuve du réel, dans la situation de travail. Or on sait aujourd'hui que, dans de multiples domaines, des savoirs réputés «acquis» en salle ne pourront jamais être exploités in vivo, par manque de pertinence, de réalisme ou d'opportunités favorables ;

- toute compétence ne se transforme pas nécessairement en performance au niveau d'organisation souhaité. D'autres circonstances, d'autres médiations sont nécessaires à cette 
transaction. Par exemple, un vendeur compétent, formé dans des conditions d'excellence, ne pourra réaliser les performances dont il est capable si le marché se révèle déprimé ou décalé par rapport à ses attentes.

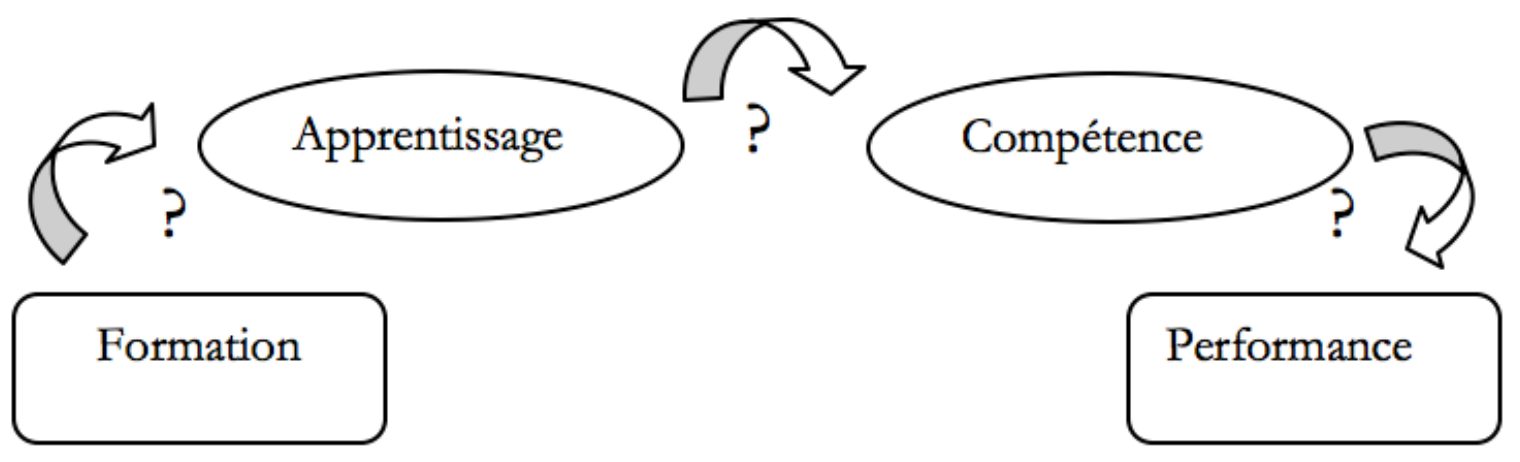

Figure 3. La triple illusion de la formation continue

Source : Carré, 2005.

Cette triple illusion donne à la formation, aux plans pédagogique, politique et social, une toutepuissance que la réalité des dynamiques individuelles, du fonctionnement social et du jeu économique vient régulièrement démentir. On a ainsi pu penser longtemps que la formation saurait régler les questions d'un autre ordre, qu'il s'agisse de résorption du chômage, de compétitivité des entreprises ou de cohésion sociale. Or la formation ne peut que participer à ces problématiques, en accompagnant le développement des compétences. La mission de la formation n'est ni de créer des emplois, ni de gagner des parts de marché. Elle est de faciliter les apprentissages qui pourront contribuer à l'atteinte de ces objectifs. Par-delà le consensus sur ce thème, cette évidence est encore loin d'être traduite dans les pratiques, malgré le développement de formules hybrides, combinant dispositif formel de formation et proximité de la situation de travail (alternance, $\mathrm{FEST}^{1}$, simulation, analyse des pratiques). Si elles rapprochent le sujet des conditions de la performance, ce qui ne peut qu'être bénéfique à l'apprentissage, ces modalités pédagogiques ne garantissent aucunement l'adhésion et l'implication du sujet concerné au projet de formation et encore moins l'atteinte des objectifs de performance visés. Le syndrome de la fabrication du projet d'autrui (Meirieu, 1996) domine encore la formation.

\section{Rendre la priorité à l'apprentissage}

Tout comme la formation initiale, et en dépit d'innovations fréquentes, la formation des adultes peine à aménager les pratiques dans le sens de la priorité à l'«apprendre ». On retrouve d'abord, parmi les causes plus ou moins anciennes de cette résistance, la prégnance du modèle scolaire de

1 FEST : formation en situation de travail. 
la transmission frontale. Houssaye, dans un ouvrage récent, souligne à quel point cette tradition pédagogique puise ses racines au plus profond de notre culture :

On ne peut s'empêcher de penser que notre pédagogie traditionnelle reste hantée par cette conception ancienne où la tradition est révélation et le cours transmission de la révélation, à l'image de ce que représentaient les Pères de l'Église au Moyen Âge. Serions-nous en présence d'une coagulation inconsciente entre la leçon, le maître, le savoir divin et Dieu lui-même ? (Houssaye, 2014, p. 210)

On évoquera également, pour illustrer la persistance de cette priorité à la transmission «descendante », la légitimité ressentie par les adultes, décideurs et responsables, à "penser la pédagogie» en experts de leur propre expérience à dominante scolaire; la persistance de modèles structuralistes de «reproduction» sociale par l'éducation (malgré les dérégulations multiples de la postmodernité) ; l'hypothèse selon laquelle la formation sert, finalement, à bien d'autres choses qu'à apprendre et, bien sûr, son inféodation, soulignée ci-dessus, à d'autres ordres de logiques et de pouvoirs sociaux, politiques et économiques, qui la dépassent ${ }^{2}$.

L'erreur pédagogique fondamentale qui obère encore le potentiel d'innovation et d'efficience des dispositifs de formation d'adultes au XXI siècle consiste donc à penser, comme hier, que l'apprentissage résulte de l'action exercée de l'extérieur par un «formateur » sur un "formé », récepteur du processus de transmission. Une seconde erreur amène à poser que la formation est clé de la performance, ce qui fait abstraction des conditions non "pédagogiques» de la compétence, puis de la performance elle-même. Ces illusions sont entretenues par l'inertie inhérente au fonctionnement des institutions d'éducation et de formation, dont l'évolution accuse toujours un retard notable sur les changements sociaux et technologiques.

Désamorcer l'erreur pédagogique et remettre la formation à sa place, c'est alors "penser à l'envers » en donnant la priorité à l'acte d'apprendre.

\section{L'apprentissage des adultes}

Pour tenter une synthèse, évidemment partielle et datée, de ce que représente la notion d'apprentissage en formation d'adultes, nous partirons d'un aperçu des apports des grandes bases théoriques, avant d'en retenir les dimensions essentielles : cognitive, conative et stratégique.

2 L'inertie de la pensée sur la formation comme action de transformation d'autrui trouve son illustration lexicale dans la traduction courante du terme de "lifelong learning " en formation tout au long de la vie. Ici encore, l'accent initial sur l'apprentissage par le sujet social (learning) est relégué aux oubliettes, au profit de l'intervention d'autrui (formation)... 
Nous conclurons en proposant, à la suite d'Evans (in Malloch, Cairns, Evans et al., 2013), la notion d'«agentivité limitée » pour exprimer l'articulation des pratiques d'apprentissage entre dispositions individuelles et facteurs contextuels.

\section{Bref rappel des grandes théorisations de l'apprentissage des adultes}

Ce tour d'horizon nécessairement allusif passera par trois étapes. Nous rappellerons d'abord le rôle essentiel joué par les grands systèmes théoriques construits depuis un siècle dans le domaine de la psychologie générale de l'apprentissage. Nous aborderons ensuite les apports de l'andragogie, ou théorie de l'apprentissage des adultes, et terminerons avec les nouveaux courants de recherche de l'apprentissage (autodirigé, transformateur, critique) en cours sur le champ spécifique à la formation des adultes. Nous chercherons dans ces rappels, nécessairement synthétiques, les fondements d'une psychopédagogie pluridimensionnelle des adultes.

\section{Les psychologies générales de l'apprentissage}

Les auteurs qui se sont penchés sur les théorisations successives de l'apprentissage depuis un siècle retiennent une demi-douzaine de "familles » théoriques ${ }^{3}$ : behaviorisme, humanisme, constructivisme, socioconstructivisme, traitement de l'information, sociocognitivisme.

Il est d'usage de débuter un tel panorama avec le behaviorisme ou théorie du conditionnement, en remontant aux travaux initiaux de Pavlov (1849-1938), puis de Thorndike et Watson. Ces recherches qui ont un intérêt aujourd'hui avant tout historique ont néanmoins mis en évidence la force des associations de stimuli dans l'apprentissage de certaines réponses comportementales. La théorie du conditionnement opérant, mise au point par Skinner (1878-1958) et ses élèves, a établi combien les conséquences des actes (renforcement, punition) influencent la probabilité de répondre aux sollicitations ultérieures du contexte. Vers le milieu du $\mathrm{Xx}^{\mathrm{e}}$ siècle, la remise en cause des postulats behavioristes a ouvert la voie d'une part à une psychologie dite « humaniste» et d'autre part à ce qu'il a été convenu d'appeler la « révolution cognitive».

L'émergence d'un courant bumaniste a été très liée à la carrière et la personnalité de Carl Rogers. Très influent au cours de la seconde moitié du $\mathrm{Xx}^{\mathrm{e}}$ siècle dans les milieux des relations humaines, de la psychothérapie et de la pédagogie, son œuvre semble aujourd'hui tomber en désuétude, sans doute à tort, du fait des excès (et des critiques) de certaines interprétations de l'orientation hâtivement nommée «non directive » qui a dominé ce courant (Carré, 2003). La prise en compte des dimensions émotionnelles, affectives et relationnelles dans les situations d'apprentissage, en lien avec le développement d'une psychologie clinique «centrée sur le

3 Voir nos sources en bibliographie. 
client », fait de Rogers l'une des figures légitimes des théories de l'apprentissage, même si les processus d'acquisition de connaissances n'ont jamais été l'objet central de ses travaux.

Sous la bannière cognitiviste peuvent s'inscrire quatre groupes théoriques: constructivisme, socioconstructivisme, théories du traitement de l'information et sociocognitivisme. Ces théories ont pour caractéristique principale de chercher à élucider les opérations mentales et fonctionnements psychologiques en se démarquant à la fois du behaviorisme, de la psychanalyse et des courants humanistes.

À partir de l'œuvre de Piaget (1896-1980), le constructivisme a durablement marqué la psychologie du développement humain et de l'intelligence, ainsi que la pédagogie dans le monde au cours de la seconde moitié du $\mathrm{XX}^{\mathrm{e}}$ siècle, ouvrant le chemin aux théories de l'activité et aux travaux des «post-piagétiens », plus tournés vers les aspects sociaux et affectifs de l'intelligence. De nombreuses critiques ont en effet été adressées aux positions piagétiennes, estimées insuffisamment tournées vers la prise en compte du contexte social et des interactions dans les apprentissages et le développement humains. Avec la découverte tardive (en 1985) des travaux du psychologue soviétique Vygotsky (1896-1934), prématurément disparu cinquante ans plus tôt et la traduction en français des travaux de Bruner (né en 1915), le relais est pris dans le dernier quart $\mathrm{du} \mathrm{XX}^{\mathrm{e}}$ siècle avec le socioconstructivisme. Toujours basées sur le postulat d'un développement cognitif par construction progressive des connaissances, les recherches de cette orientation mettent l'accent, dans la tradition initiée par les psychologues marxistes en URSS, et certains auteurs français comme Wallon et Meyerson, sur la dimension socio-historique et culturelle de la construction des connaissances ${ }^{4}$. Plus récemment, les théoriciens du traitement de l'information (information processing theories), parfois intitulé connexionisme, étudient les séquençages et modes d'exécution des évènements cognitifs, en particulier (mais non uniquement) en situation de laboratoire. Inscrites dans le champ vaste des sciences cognitives, ces recherches se situent à la croisée de la psychologie, de l'intelligence artificielle et de la biologie.

Enfin, une place à part, dans la double continuité du behaviorisme et du cognitivisme, doit être accordée à la théorie sociocognitive de Bandura (2003), parce qu'elle combine certaines des influences précédentes à travers le schéma intégratif dit de la "causalité triadique réciproque ». Pour cet auteur et ses collègues (Schunk, 2000; Zimmerman \& Schunk, 2003), le fonctionnement humain (et, partant, l'apprentissage) est conçu à partir d'une série d'interactions réciproques entre dispositions personnelles, déterminants contextuels et comportements

4 Voir par exemple, pour la francophonie, Pastré, in Bourgeois \& Chapelle (2006), et dans une dimension plus internationale, Engeström, in Malloch, Cairnes, Evans et al. (2013). 
effectifs. Dans ce modèle, l'apprentissage est vu comme un processus proactif et émergent combinant observation et modelage (apprentissage «vicariant»), construction de buts, sentiment d'efficacité personnelle, pratiques d'autorégulation. Ce dernier point est à la fois central pour notre propos et d'une actualité évidente. Pour Bandura, l'apprentissage est un processus «agentique », c'est-à-dire porté par le pouvoir d'agir du sujet : «le contenu de la plupart des manuels est périssable, mais les ressources de l'autodirection seront utiles de tout temps » (in Zimmerman \& Schunk, 2003, p. 432).

Les théories de l'apprentissage portées par ces différentes psychologies tout au long du $\mathrm{XX}^{\mathrm{e}}$ siècle ont, en quelque sorte, construit par sédimentation progressive un corpus de connaissances sur «comment apprend l'adulte» dont chacune porte l'accent sur une dimension spécifique du processus, qu'il s'agisse de l'importance des renforcements (behaviorisme), de la relation d'aide (humanisme), du rôle de l'activité (constructivisme), de celui des interactions sociales (socioconstructivisme), de l'agentivité humaine (sociocognitivisme). Venus des horizons de la pratique et du champ spécifique de la formation des adultes, différents courants parfois dits «andragogiques» viennent compléter et renforcer sur le versant pédagogique l'essor d'une " psychopédagogie des adultes » en émergence.

\section{Andragogie et théories de l'apprentissage des adultes}

Même si les figures d'auteurs francophones influents traversent l'histoire des idées sur le champ de la formation des adultes, que ce soit dans le domaine du travail avec Schwartz ou des loisirs et de la culture avec Dumazedier, pour ne citer qu'eux, on trouve peu de théorisation systématique des spécificités de l'apprentissage adulte produits dans cette langue ${ }^{5}$. Trois auteurs nordaméricains sont aujourd'hui reconnus comme les «pères fondateurs » des premières théories des spécificités de l'apprentissage adulte: Houle, Tough et Knowles. Leurs travaux initiaux, produits au cours des années 1960-1970 ont donné lieu à de nombreuses variations, initialement réunies autour de la bannière de l'andragogie, puis de divers courants dont nous retiendrons avec Merriam et Caffarella (1999) comme les principaux ceux de l'apprentissage autodirigé (selfdirected learning), transformateur (transformative learning), et de la théorie critique (critical theory).

La notion d'andragogie ou "art et science d'aider les adultes à apprendre » introduite par Knowles en 1968 se donne pour objet l'étude des spécificités de l'apprentissage adulte par rapport à celui des enfants. Cet auteur dénombre alors cinq caractéristiques différenciatrices,

5 Il convient de mentionner ici la nuance à cette règle que fournit l'essor, à la fin du siècle dernier, des approches biographiques de la formation dont les fondements variés se rattachent à des perspectives théoriques diverses. 
autour des transformations caractéristiques de l'âge adulte dans les domaines de l'autonomie, de l'expérience, des rôles sociaux, des perspectives temporelles et des motivations. Il en tire, sur un plan pédagogique, une série de recommandations tournées vers les notions de contrat, de facilitation et d'aide à l'autoformation. Plusieurs critiques ont été adressées à l'orientation andragogique, par-delà la seule dimension terminologique ${ }^{6}$, tant dans son volet théorique que dans ses présupposés opposant de façon radicale l'andragogie à la pédagogie. Néanmoins, cet ensemble plus pédagogique que psychologique à proprement parler a donné aux éducateurs d'adultes du monde entier une forme d'identité partagée et ouvert la voie à de nouveaux courants aujourd'hui fortement développés dans le domaine.

Le courant de l'apprentissage autodirigé assume depuis le début des années 1980 une large part d'héritage de la pensée de Knowles qui avait largement contribué à la diffusion de ce thème tant sur le plan de la recherche que sur celui des pratiques. D'abord réuni sous la houlette de Long, puis de Guglielmino, le symposium annuel sur l'apprentissage autodirigé, au cours de ses trente éditions à ce jour, a largement contribué par plusieurs centaines de publications à faire de ce thème l'un des plus vivaces de la recherche nord-américaine et, dans une certaine mesure, internationale, sur la formation des adultes. Les auteurs se sont attachés à dégager les dimensions constitutives de l'apprentissage autodirigé qui recouvrent autant de catégories d'analyse: psychologie du sujet apprenant, mesure de l'autodirection comme variable psychométrique, dimensions sociales et groupales de l'apprentissage, dimensions pédagogiques et technologiques de la facilitation.

La notion d'apprentissage transformateur, proposée par Mezirow en 1978, s'inscrit dans le double héritage de la pensée de Knowles et de celle l'éducateur latino-américain Freire. Le concept d'expérience, transformatrice pour le premier, émancipatrice pour le second, est au cœur de ce courant, qui a donné lieu à un foisonnement de recherches et de développements théoriques depuis la dernière décennie du XX ${ }^{\mathrm{e}}$ siècle (Merriam \& Caffarella, 1999). Pour Mezirow, l'expérience de vie, d'autant plus conséquente chez l'adulte, donne lieu à une recherche de sens susceptible de générer des transformations de perspective qui, à leur tour, apportent la possibilité de changements plus ou moins radicaux dans l'activité et la conduite de sa vie à l'occasion de transitions biographiques multiples. La praxis est ainsi définie comme un cycle permanent de réflexion, de modification des représentations et de réflexion critique sur l'action. La dimension critique est plus présente encore dans la philosophie pédagogique de Freire, à travers les concepts de conscientisation, de dialogue émancipateur et d'autonomie qui ont fourni les bases des conceptions actuelles de l'empowerment. Les travaux de cet auteur ont eu une

6 Le radical andro- caractérisant sur le plan étymologique la masculinité. 
résonance internationale remarquable depuis les années 1970. Ils ont fourni aux instances supranationales et aux mouvements d'éducation populaire des pays les moins développés un modèle de compréhension et d'intervention qui ouvrait les voies d'un changement social radical grâce à l'éducation des adultes, l'alphabétisation et l'exercice de la pensée critique sur les conditions sociales d'existence des plus défavorisés. On trouvera aisément les traces de ce courant politiquement engagé dans les travaux d'orientation postmoderne sur l'éducation des adultes dans une perspective "radicale », orientée vers la justice sociale et la lutte contre les inégalités sociales, de race ou de genre.

\section{Vers une synthèse ?}

En définitive, la question des spécificités de l’apprentissage adulte, pour être abordée de façon heuristique et efficiente, implique un double mouvement.

D’une part, le dépassement des oppositions de courants pour dégager, par-delà les postures idéologiques et les postulats rigides, les apports singuliers de chaque théorisation à l'intelligibilité des dynamiques d'acquisition de savoirs et de développement de compétences. De ce point de vue, tant les psychologies générales que les théorisations spécifiques identifiées cidessus peuvent former les fondements d'une conception large et ouverte des continuités et des spécificités de l'apprentissage de l'enfance à l'âge adulte, ou, plus précisément, de la situation scolaire aux situations de formation post-scolaire, dans la logique actuelle de l'apprentissage tout au long de la vie.

D'autre part, et de façon indissociable, la compréhension des faits de formation des adultes passe sans doute par un processus de différenciation des niveaux d'analyse, pour autant que, dans les termes de Desjeux (2004), le réel change en fonction de l'échelle à laquelle on l'observe. Les théories concurrentes peuvent ainsi s'articuler selon qu'elles sont ciblées sur un niveau micro (individuel), méso (groupal) ou macro (culturel-politique), évitant par là les risques opposés du psychologisme ou, à l'inverse, du sociologisme dans l'analyse des dimensions plurielles du monde et de l'individu (Lahire, 2013).

\section{Un postulat et trois paramètres essentiels}

Les théories de l'apprentissage sont donc multiples dans la psychologie générale et dans le domaine de la formation des adultes, comme l'attestent les déclinaisons variées des grands courants mentionnés ici, et donnent lieu à peu de synthèses intégratives. Les références bibliographiques citées en fin d'article renvoient à de multiples concepts issus de ces courants de recherche variés, parfois complémentaires, souvent opposés dans leurs présupposés méthodologiques et épistémologiques. On retiendra cependant une certaine convergence autour des principes de base suivants. 


\section{L'apprentissage, phénomène individuel et social}

Un postulat aujourd'hui partagé par la recherche sur les apprentissages des adultes est que ceuxci sont nécessairement, et indissociablement, des processus à la fois individuels et sociaux. En somme, on apprend toujours seul, mais jamais sans les autres. Les nuances entre les théories tiennent en grande partie aux poids respectifs accordés d'une part à l'agentivité du sujet et aux facteurs dispositionnels (biographie, expérience, motivation, capacités, attitudes) et d'autre part à la structure de la situation (contexte, environnement, affordances, ressources). Il est aujourd'hui acquis que les manifestations comportementales en jeu dans l'apprentissage (qu'on les dénomme, selon la culture professionnelle de référence, pratiques, activités ou performances) s'organisent au confluent des dispositions et des situations, dans une interaction dialectique entre facteurs personnels et contextuels traversée par la dimension sociale du fonctionnement humain, pour autant que, comme l'écrivait Wallon, séparer l'individu de la société, ce serait « lui décortiquer le cerveau ».

Sur la base de ce postulat, trois paramètres à coefficients variables apparaissent dans l'équation complexe de l'apprentissage : le rôle des préconceptions et des connaissances préalables à l'apprentissage, l'importance des facteurs conatifs (attitudes, motivation, volition), la place des stratégies d'autorégulation et de la métacognition.

\section{Les facteurs cognitifs : le rôle des connaissances antérieures}

Le rôle des connaissances préalables de l'apprenant dans la conduite de ses apprentissages est un facteur commun de la plupart des conceptions psychopédagogiques, qu'il s'agisse des renforcements de l'expérience passée dans l'optique behavioriste, des représentations antérieures, schèmes acquis ou préconceptions de l'apprenant selon les entrées cognitivistes ou de la place des expériences biographiques dans les émotions pour la psychologie humaniste. Loin de la vision de l'apprenant comme une «table rase», les théories s'accordent pour souligner la singularité des représentations, des dispositions et des schémas de pensée, justes et/ou erronés, avec lesquels le sujet social aborde un savoir. L'intégration de nouveaux concepts, d'habiletés ou d'attitudes inédites par l'apprenant exige donc en premier lieu la mise au jour de ces conceptions, voire leur déconstruction, avant que l'assimilation des données nouvelles soit possible, et ce d'autant plus que les anciennes conceptions sont solidement ancrées. Il a régulièrement été établi que les connaissances préalables sont la variable la plus influente dans les performances académiques (De Corte, in OCDE, 2010).

\section{Les facteurs conatifs : attitudes, motivation, volition}

On considère aujourd'hui dans leur spécificité les facteurs conatifs comme concernant le « choix et l'orientation des conduites », à côté des facteurs " purement » cognitifs (opérations mentales) 
et affectifs (émotions), dans l'ensemble interconnecté de ces trois fonctions psychiques fondamentales (Carré \& Fenouillet, 2009).

Concernant la motivation, après une période de recul théorique dans les années 1970-1980, on observe une explosion des travaux empiriques et conceptuels sur cet objet, d'abord en Amérique du Nord, puis en Europe depuis l'orée du troisième millénaire. Fenouillet a ainsi pu dégager plus de cent théories de la motivation dont une majorité concerne les processus d'apprentissage (Carré \& Fenouillet, 2009). Il ne saurait être question d'en faire ici état. Rappelons simplement la variété des concepts invoqués, qu'il s'agisse du rôle des croyances motivationnelles (sentiment d'efficacité, attente de résultats, croyances sur soi, théories du self...), de la construction et du rôle des buts, de la valeur perçue des motifs, de l'autodétermination, et des notions connexes de dynamique identitaire et d'estime de soi. Ces concepts à la valeur empirique et heuristique aujourd'hui reconnue interviennent puissamment dans la compréhension des apprentissages adultes. La relation entre motivation et formation fait l'objet de travaux empiriques et théoriques de plus en plus nombreux aujourd'hui ${ }^{7}$.

À la périphérie des processus motivationnels pour certains auteurs, en leur cœur pour d'autres, l'étude des facteurs conatifs concerne également, en amont de l'action, la question des attitudes et, en aval, celle de la volition. Dans la phase prédécisionnelle de la motivation, les attitudes sont des dispositions cognitives, affectives et conatives, qui peuvent se transformer en motifs d'engagement dans l'action. À l'heure où les pouvoirs publics, les entreprises et les instances supranationales encouragent en un bel ensemble les citoyens à adopter une attitude "apprenante », la thématique du rapport individuel à la formation sous l'angle des attitudes revêt une importance cruciale, en particulier autour de la question du lien entre attitudes et action, aujourd'hui objet de controverses en psychologie sociale (Carré, 2005). Enfin, la phase post-décisionnelle de la motivation transforme celle-ci en volition, où elle se combine avec l'adoption de stratégies d'action.

\section{Les stratégies d'autorégulation}

Très lié au concept de volition, le thème des stratégies d'autorégulation fonde la troisième constante des processus d'apprentissage, à la lumière de travaux récents qui mettent en lumière l'impact des connaissances métacognitives et des habitudes de travail sur l'efficacité des apprentissages. Selon Cosnefroy :

7 Ici encore, nous ne pouvons que renvoyer le lecteur aux références, par exemple, de Bourgeois, in Bourgeois \& Chapelle (2006) ; Carré \& Fenouillet, in Carré \& Caspar (2011) ; Bourgeois, in Carré \& Fenouillet (2009). 
Parler d'apprentissage autorégulé, c'est mettre l'accent sur la participation active de l'apprenant au processus d'apprentissage [...]. De nombreux travaux convergent pour souligner que la capacité à s'autoréguler est une variable décisive pour améliorer la réussite dans les apprentissages et pour comprendre la différence de réussite entre les apprenants (2011, p. 10).

L'autorégulation (ou apprentissage autorégulé) renvoie aux processus par lesquels les apprenants gèrent systématiquement leurs pensées, leurs émotions et leurs actions en vue d'atteindre leurs objectifs d'apprentissage. Ces processus combinent l'attention, l'entraînement, l'utilisation de stratégies et le suivi des progrès avec les croyances d'efficacité et les perceptions de la valeur des efforts consentis. L'autorégulation a donc deux dimensions : motivationnelle d'une part et comportementale (ou stratégique) d'autre part (Schunk, 2000).

En conclusion de l'ouvrage de l'OCDE (2010) sur la question "comment apprend-on ?", De Corte résume les conditions de l'apprentissage efficace, sur la base des données actuelles de la recherche, par les qualificatifs suivants : il sera «constructif, autorégulé, situé et collaboratif ». Ces termes soulignent certains des vecteurs majeurs de la réussite pour apprendre, en reprenant les lignes de force des théories mentionnées ci-dessus.

\section{Apprendre : une pratique agentique limitée}

L'ensemble des données de recherche ébauchées dans cet article confirme la priorité à accorder, dans la compréhension et l'optimisation des apprentissages adultes, à l'activité de l'apprenant, que ce soit sous l'angle des interactions sociales, des connaissances préalables, des motivations, des pratiques d'autorégulation. Le parti pris de priorité à l'apprentissage trouve sa justification dans la recherche actuelle, qui souligne l'agentivité, ce pouvoir d'agir du sujet, comme caractéristique essentielle de l'efficacité (OCDE, 2010 ; Schunk, 2000 ; Bourgeois \& Chapelle, 2006).

Toutefois, comme le souligne Evans, cette agentivité ne peut se déployer qu'au cœur d'un réseau d'influences complexes, sociales et environnementales, qui donnent au contexte d'apprentissage un rôle également déterminant. Pour Evans :

La façon dont les adultes apprennent dans et par le travail est enracinée dans des trajectoires éducatives et leur intrication complexe avec des institutions sociales (marché du travail, organisation, communauté) et des rôles sociaux (d'employé, de citoyen, familiaux) à différentes étapes du cycle de vie (in Malloch, Cairns, Evans et al., 2013, p. 356). 
Les théories contemporaines de l'apprentissage au travail reconnaissent toutes les rôles respectifs de l'agentivité et de la structure, mais diffèrent entre elles sur les poids à accorder à l'une et à l'autre. L'agentivité existe donc, mais n'opère pas « hors sol» :

Les croyances des gens en leurs capacités de changer leurs situations grâce à leurs propres efforts sont significatives pour le développement de leurs compétences au travail et audelà [...]. La capacité de traduire ces croyances en actes se construit plus qu'elle n'est acquise et ces capabilités sont limitées par des contraintes qui peuvent être desserrées (Evans, in Malloch, Cairns, Evans et al., 2013, p. 356).

L'agentivité du sujet est donc première dans les processus d'apprentissage, mais il s'agit d'une agentivité limitée par le tissu social, organisationnel et politique qui l'enserre. La formation peut contribuer à transformer cette contrainte en opportunité et jouer un rôle facilitateur.

\section{Faciliter les apprentissages des adultes}

\section{Une pédagogie de la facilitation}

Plusieurs caractéristiques essentielles ressortent de cette brève analyse : d'un point de vue psychopédagogique, l'apprentissage efficient sera inscrit dans l'articulation des dispositions individuelles et des facteurs de contexte; il sera construit à partir des connaissances préalables du sujet, motivé et autorégulé. À partir de ces prémisses, une pédagogie de la «facilitation » aura une triple mission d'invitation à l'activité d'apprentissage, d'accompagnement et d'évaluation, formative en particulier, du travail de l'apprenant. Inversée, ascendante, participative, elle se fera côte à côte plutôt que face-à-face, dans une posture d'aide méthodologique, relationnelle et didactique que la notion de «soutien à l'autonomie » (autonomy support) proposée par Reeve (2009) capture bien. Une autre illustration des principes pédagogiques que l'on peut extraire de l'analyse psychopédagogique des lois de l'apprentissage peut être trouvée dans les trois principes d'autoproduction, autodirection et collaboration proposés par Poisson (in Carré, Moisan \& Poisson, 2010).

Enfin, l'efficience d'un dispositif pédagogique se mesurera à l'aune de son articulation avec les dispositions des individus : connaissances préalables, dynamiques motivationnelles, capabilités (en particulier d'autorégulation). C'est le rôle de l'ingénierie pédagogique des dispositifs de multiplier les interfaces entre la structure et le sujet, que ce soit en amont (information, accueil, diagnostics préalables, orientation, contractualisation...), pendant l'apprentissage (personnalisation, aide à l'autorégulation, feedback, ressources, organisation, technologie...) ou après celui-ci (appui au transfert, évaluation sommative). Ce sont là les principes ambitieux d'une pédagogie de la facilitation que le recours aux ressources numériques d'une part, à 
l'autorégulation des apprenants ensuite, et à la «maîtrise d'usage ${ }^{8}$ enfin, rend réalistes si l'on souhaite résolument passer d'une culture de la formation à une culture de l'apprenance.

Notons, pour terminer, que la facilitation de l'apprentissage adulte, en tant que processus individuel socialement situé de transformation durable de représentations, d'habiletés et d'attitudes, ne borne pas son périmètre aux situations de formation en milieu éducatif, qu'il soit formel (cours, stages, séminaires) ou semi-formel (e-learning, formations "ouvertes»). Elle pourra se déployer dans l'accompagnement des projets d'autoformation en milieu informel, à travers des formes « légères » et, par définition, non instituées d'accompagnement, de parrainage, de coaching ou de conseil. Les formes que prendra la posture de facilitation dans ses dimensions relationnelle, méthodologique ou didactique varieront en fonction de l'articulation recherchée entre dispositions des apprenants et possibilités du contexte d'apprentissage. La recherche sur les apprentissages des adultes dans la «société cognitive» est directement interpellée sur ce point.

\section{Pour une nouvelle psychopédagogie des adultes}

Le domaine de la formation des adultes est un champ de recherches récent, principalement inscrit dans le cadre lui-même encore jeune des sciences de l'éducation. Les analyses qui y sont menées souffrent de multiples handicaps et confusions. Sur le plan pratique, l'expertise y est rendue délicate par la familiarité que tout acteur adulte entretient avec l'apprentissage en fonction de sa propre expérience (scolaire, familiale, universitaire, en formation continue), donnant aux préconceptions singulières force d'évidence. Sur un plan conceptuel, discours et recherches ne clarifient pas toujours le(s) niveau(x) d'analyse mobilisé(s), ouvrant sur un risque de myopie épistémologique et menant vers les trois écueils du psychologisme, du sociologisme ou du pédagogisme. Le domaine souffre également de fréquentes généralisations à partir d'un niveau d'analyse ciblé sur l'une des dimensions de ce que l'on nomme «la» formation. Chercheurs et praticiens du champ ont sans doute intérêt de ce point de vue à spécifier quelle échelle d'observation ils utilisent, et quel niveau d'organisation ils s'attachent à défricher, quitte à travailler aux interfaces entre deux niveaux. Ce qui est le cas de la démarche psychopédagogique proposée dans cet article.

L'approche psychopédagogique classique concerne l'analyse «biunivoque » des rapports entre investigation psychologique et action éducative (Léon, 1971). L’usage du terme est resté discret en formation d'adultes, du fait d'une part des rapports institutionnels complexes entre

$8 \mathrm{La}$ notion de maîtrise d'usage, issue de l'urbanisme, traduit la proposition d'intégrer une représentation des usagers dans les différentes phases de conduite d'un projet, particulièrement lors de la conception, dans une démarche d'empowerment. Voir Vulbeau, 2010. 
psychologie et sciences de l'éducation, d'autre part de son import dans le domaine de la formation des enseignants, où il a longtemps été assimilé à une philosophie pédagogique. La constitution en émergence des fondements théoriques des conditions de l'apprentissage adulte requiert pourtant une double approche, en termes de psychologie générale de l'apprentissage d'une part, de facilitation pédagogique d'autre part, dont le concept de psychopédagogie permet de saisir les effets d'interaction.

Ce sont bien des conceptions psychopédagogiques de la formation des adultes qui traversent aujourd'hui l'ingénierie pédagogique, l'analyse de l'activité, l'analyse des pratiques et de multiples formes d'innovation. Ces pratiques sont toujours adossées à des conceptions implicites ou explicites de ce qu'apprendre veut dire. Corrélativement, l'analyse des effets d'une formation est nécessairement imprégnée de telles conceptions, plus ou moins conscientisées, plus ou moins justes, de l'efficience psychologique de la pédagogie. Les relations entre psychologie des apprentissages et pédagogie des adultes sont toujours au cœur des débats sur l'efficience de la formation.

Un paradigme psychopédagogique renouvelé semble donc nécessaire à la construction progressive d'un corpus scientifique sur le «pourquoi » et le «comment» de l'apprentissage adulte, ingrédient réputé essentiel du développement économique, social et humain au XXI siècle. Un cadre théorique triadique tel que celui proposé par la théorie sociocognitive (Bandura, 2003), permettant l'analyse des interactions réciproques entre pratiques, dispositions personnelles et facteurs de contexte, semble une plateforme adaptée au projet de refondation d'une psychopédagogie des adultes «ouverte» sur les disciplines connexes participant à la compréhension des faits de formation (économie, histoire, sociologie, informationcommunication, anthropologie). Un tel cadre tripolaire «ouvert» trouverait écho à la fois dans les fondements historiques de la psychopédagogie des adultes (Léon, 1971, p. 76) et dans les bases assignées par Lahire dans son ouvrage récent sur l'unité des sciences sociales (Lahire, 2013).

Des racines et un horizon en somme, pour une nouvelle psychopédagogie des adultes.

\section{Bibliographie}

BANDURA A. (2003). Auto-efficacité. Le sentiment d'efficacité personnelle. Bruxelles : De Boeck.

BERBAUM J. (1994). Apprentissage et formation. Paris : PUF.

BOURGEOIS E. \& CHAPELLE G. (dir.) (2006). Apprendre et faire apprendre. Paris : PUF. 
BROUGÈRE G. \& ULMANN A.-L. (dir.) (2009). Apprendre de la vie quotidienne. Paris : PUF.

CARRÉ P. (2003). «Rogers, ou de l'autodirection ». In Collectif « Savoirs et rapport au savoir », Autobiographie de Carl Rogers. Lectures plurielles. Paris : L’Harmattan, p. 223240.

CARRÉ P. (2005). L'apprenance. Vers un nouveau rapport au savoir. Paris : Dunod.

CARRÉ P. \& CASPAR P. (dir.) (2011). Traité des sciences et techniques de la formation. Paris : Dunod.

CARRÉ P. \& CHARBONNIER O. (dir.) (2003). Les apprentissages professionnels informels. Paris : L'Harmattan.

CARRÉ P. \& FENOUILLET F. (dir.) (2009). Traité de psychologie de la motivation. Paris : Dunod.

CARRÉ P., MOISAN A. \& POISSON D. (dir.) (2010). L'autoformation. Perspectives de recherche. Paris : PUF.

COSNEFROY L. (2011). L'apprentissage autorégulé. Entre cognition et motivation.

Grenoble : Presses universitaires de Grenoble.

DESJEUX D. (2004). Les sciences sociales. Paris : PUF.

ENLART S. (2013). «Financement de la formation : l'imputabilité devrait être abordée sous l'angle de l'efficacité pédagogique ». Dépêche $A E F, \mathrm{n}^{\circ} 179825$ du 27 février 2013.

HIEMSTRA R. \& CARRÉ P. (dir.) (2013). A Feast of Learning. International Perspectives on Adult Learning and Change. Charlotte (États-Unis) : IAP.

HOUSSAYE J. (2014). La pédagogie traditionnelle. Une histoire de la pédagogie. Paris : Fabert.

LAHIRE B. (2013). Monde pluriel. Penser l'unité des sciences sociales. Paris : Éd. du Seuil.

LAMEUL G., JÉZÉGOU A. \& TROLLAT A.-F. (dir.) (2009). Articuler dispositifs de formation et dispositions des apprenants. Lyon : Chronique Sociale.

LÉON A. (1971). Psychopédagogie des adultes. Paris : PUF.

MALLOCH M., CAIRNS L., EVANS K. \& O'CONNOR B. (dir.) (2013). The SAGE Handbook of Workplace Learning. Londres : SAGE.

MEIRIEU P. (1996). Frankenstein pédagogue. Paris : ESF.

MERRIAM S. \& CAFFARELLA R. (1999). Learning in Adulthood. San Francisco : Jossey-Bass. 
OCDE (2010). Comment apprend-on? La recherche au service de la pratique. Paris : Éd. de l'OCDE.

NAGELS M. \& CARRÉ P. (dir.) (2014). Apprendre par soi-même aujourd'bui. Les nouvelles modalités de l'autoformation dans la société contemporaine. Paris : Bibliothèque publique d'information.

RAYNAL F. \& RIEUNIER A. (2010). Pédagogie, dictionnaire des concepts-clés. Apprentissage, formation, psychologie cognitive. Issy : Éd. ESF.

REEVE J. (2009). «Why Teachers Adopt a Controlling Motivating Style Towards Students and How They Can Become More Autonomy Supportive ». Educational Psychologist, $\mathrm{n}^{\circ}$ 44(3), p. 159-175.

SCHUNK D. (2000). Learning Theories. An Educational Perspective. Upper Saddle River (États-Unis) : Prentice Hall

VERMERSCH P. (2012). Explicitation et phénoménologie. Paris : PUF.

VULBEAU A. (dir.) (2010). Les Cabiers de l'ED 139. Nanterre : Université Paris-Ouest, p. 9-70.

ZIMMERMAN B. \& SCHUNK D. (dir.) (2003). Educational Psychology. A Century of Contributions. Mahwah (États-Unis) : LEA. 